\title{
Overview of the gamma reaction history diagnostic for the National Ignition Facility (NIF)
}

R. M. Malone, B. C. Cox, S. C. Evans, B. C. Frogget, H. W. Herrmann, M. I. Kaufman, Y. H. Kim, K. D. McGillivray, J. M. Mack, M. J. Palagi, W. Stoeffl, A. Tibbitts, T. W. Tunnell and C. S. Young

May 17, 2010

International Optical Design Conference on the GRH Jackson Hole, WY, United States June 13-17, 2010 


\section{Disclaimer}

This document was prepared as an account of work sponsored by an agency of the United States government. Neither the United States government nor Lawrence Livermore National Security, LLC, nor any of their employees makes any warranty, expressed or implied, or assumes any legal liability or responsibility for the accuracy, completeness, or usefulness of any information, apparatus, product, or process disclosed, or represents that its use would not infringe privately owned rights. Reference herein to any specific commercial product, process, or service by trade name, trademark, manufacturer, or otherwise does not necessarily constitute or imply its endorsement, recommendation, or favoring by the United States government or Lawrence Livermore National Security, LLC. The views and opinions of authors expressed herein do not necessarily state or reflect those of the United States government or Lawrence Livermore National Security, LLC, and shall not be used for advertising or product endorsement purposes. 


\title{
Overview of the gamma reaction history diagnostic for the National Ignition Facility (NIF)
}

\author{
Robert M. Malone ${ }^{1}$, Brian C. Cox ${ }^{1}$, Scott C. Evans ${ }^{2}$, Brent C. Frogget ${ }^{1}$, Hans W. Herrmann ${ }^{2}$, \\ Morris I. Kaufman', Yong H. Kim², Kevin D. McGillivray ${ }^{3}$, Joseph M. Mack², Martin J. \\ Palagi $^{3}$, Wolfgang Stoeffl ${ }^{4}$, Aric Tibbitts ${ }^{1}$, Thomas W. Tunnell ${ }^{1}$, Carl S. Young ${ }^{2}$ \\ ${ }^{1}$ National Security Technologies, LLC, P.O. Box 809, Los Alamos, New Mexico 87544, USA \\ ${ }^{2}$ Los Alamos National Laboratory, P.O. Box 1663, M/S \#526, Los Alamos, New Mexico, 87545, USA \\ ${ }^{3}$ National Security Technologies, LLC, P.O. Box 98521, Las Vegas, Nevada 89193, USA \\ ${ }^{4}$ Lawrence Livermore National Laboratory, P.O. Box 808, Livermore, California 94551, USA
}

\begin{abstract}
The National Ignition Facility (NIF) has a need for measuring gamma radiation as part of a nuclear diagnostic program. A new gamma-detection diagnostic uses $90^{\circ}$ off-axis parabolic mirrors to relay Cherenkov light from a volume of pressurized gas. This nonimaging optical system has the high-speed detector placed at a stop position with the Cherenkov light delayed until after the prompt gammas have passed through the detector. Because of the wavelength range $(250$ to $700 \mathrm{~nm}$ ), the optical element surface finish was a key design constraint. A cluster of four channels (each set to a different gas pressure) will collect the time histories for different energy ranges of gammas.
\end{abstract}

Keywords: gamma ray detection, National Ignition Facility, NIF, Cherenkov, non-imaging optics, off-axis parabolas

\section{INTRODUCTION}

The Lawrence Livermore National Laboratory's National Ignition Facility (NIF) is the world's largest and most powerful laser system for inertial confinement fusion (ICF) and experiments studying high energy density (HED) science. The long-term goal of attaining fusion ignition and burn at NIF is rapidly approaching. The technology challenge is to create an inexhaustible supply of clean, carbon-free energy. This optical instrument was developed to monitor the fusion process.

Figure 1 is a cartoon layout of the NIF vacuum chamber. Two out of the 192 laser beams (shown in red) enter the chamber from the bottom left. Diagnostics are mounted onto external ports of this vacuum chamber. The pencil-erasersized cylinder, shown in Figure 1, contains a tiny spherical target filled with deuterium and tritium, two isotopes of hydrogen. Inside the cylinder, the laser energy is converted to x-rays at the gold wall. The x-rays heat and compress the fuel until it reaches temperatures of more than 200 million degrees Fahrenheit and pressures billion times greater than Earth's atmospheric pressure. The rapid compression of the fuel capsule forces the hydrogen nuclei to fuse and release many times more energy than the laser energy that was required to initiate the reaction.

The deuterium-tritium (DT) fusion reaction is the most efficient reaction known in terms of energy released. The energy released is related to Einstein's mass-energy equivalence equation: $\mathrm{E}=\mathrm{mc}^{2}$. The ratio between the energy released during the fusion process to the kinetic energy needed to fuse the deuterium nucleus to the tritium nucleus is 450 . This value is a theoretical limit. The NIF break even goal is to get above 1. Figure 2 shows the products of this fusion reaction. Most of the energy is released with high-speed neutrons. But for every 100,000 neutrons released, one gamma is also released from a different reaction branch. Neutrons have mass, and their energy values can vary; therefore, they Email: malonerm@nv.doe.gov

This work performed under the auspices of the

U.S. Department of Energy by Lawrence Livermore

National Laboratory under Contract DE-AC52-07NA27344. 


\section{DOE/NV/25946-XXX}

LA-UR 10-XXXX

arrive at a neutron detector at different times. However, all gammas, regardless of their energy values, travel at the speed of light. If you measure the reaction rate of the gamma particles, very precise knowledge of the reaction history is obtained. The problem to overcome is the low numbers of gammas. 


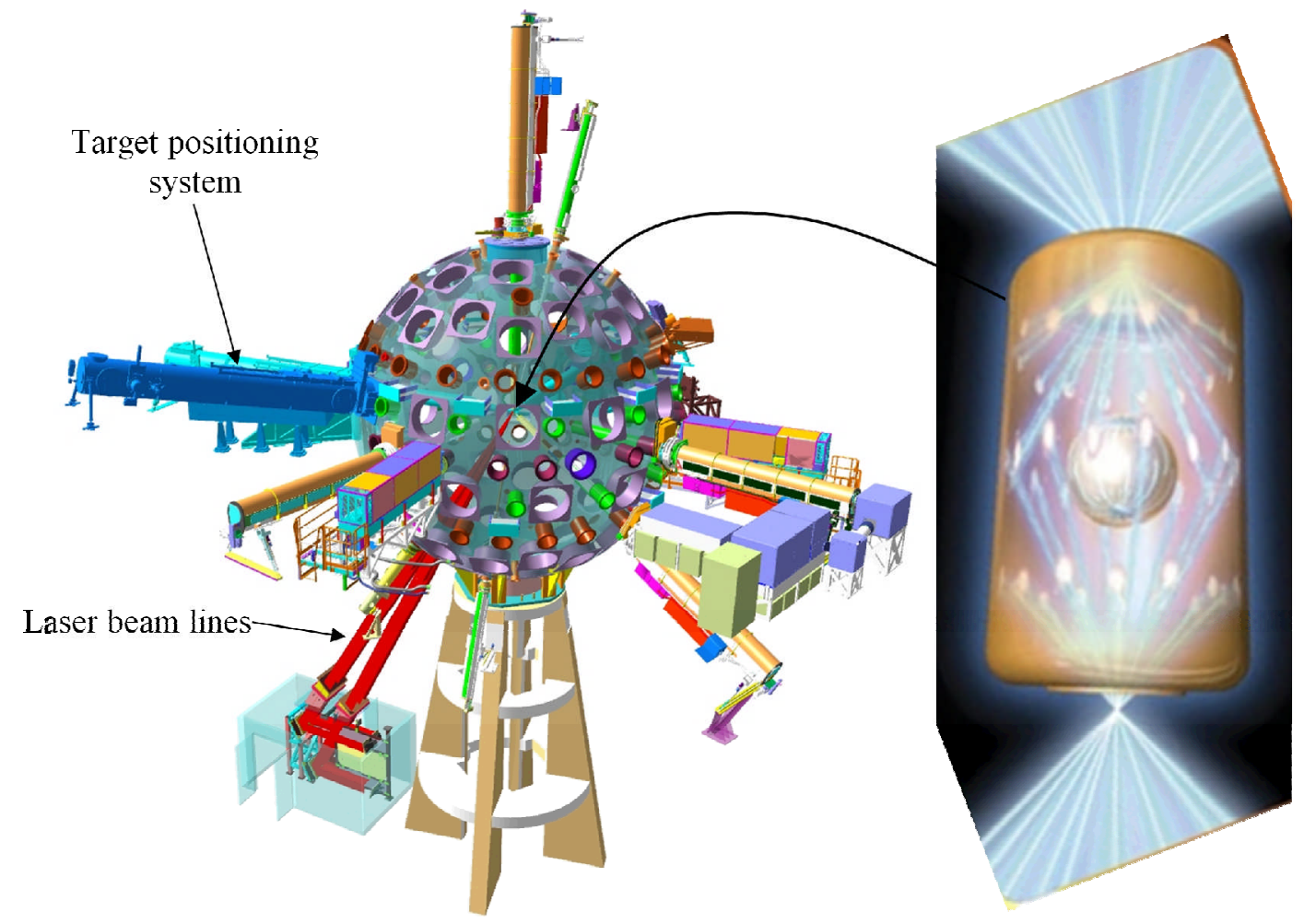

Fig. 1.The NIF target chamber has an inside diameter of 10 meters. All 192 lasers are aimed at the walls of a gold cylinder (right insert) where they are converted to x-rays. The x-rays apply the kinetic energy to cause the spherical ball of fuel to implode.

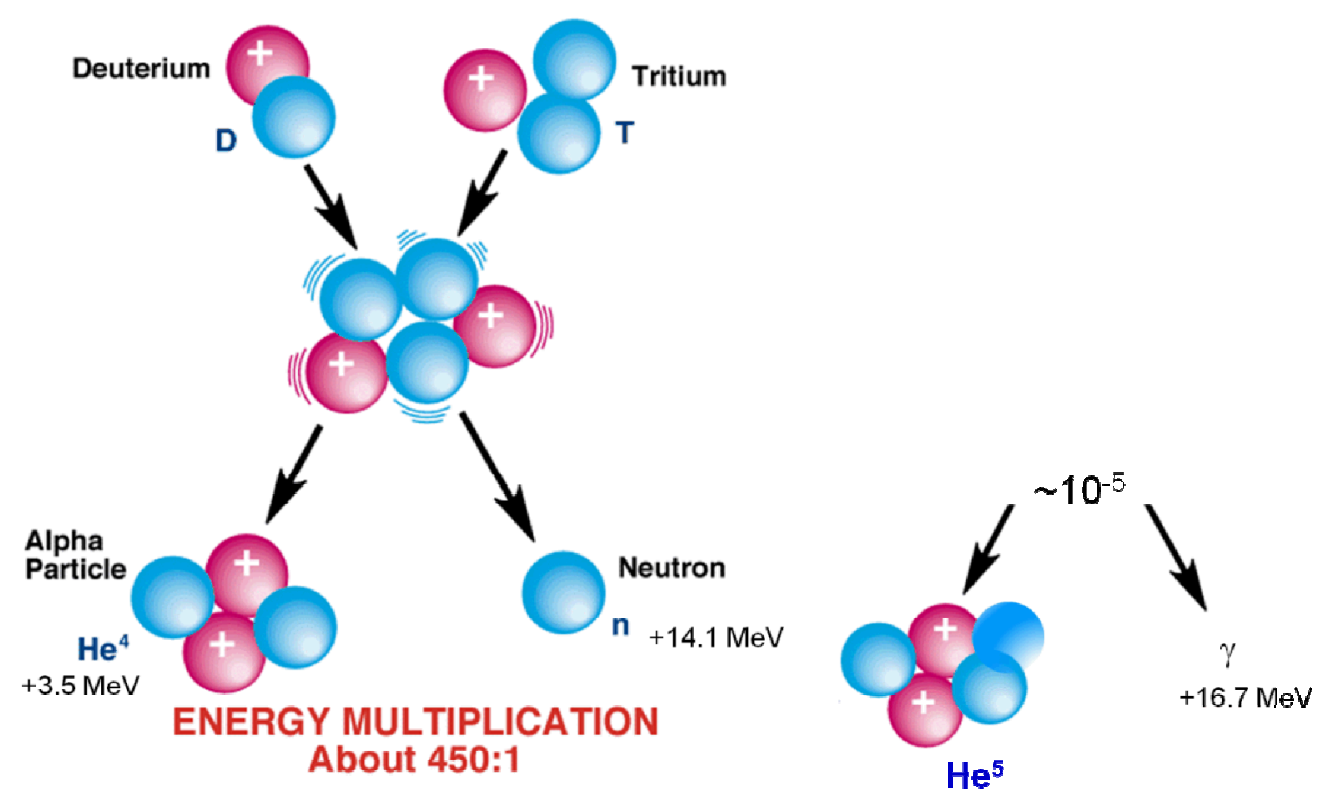

Fig. 2.The deuterium-tritium fusion reaction. $2 \mathrm{eV}$ is the energy of a red photon. A $16.7 \mathrm{MeV}$ gamma is a photon with millions of times the energy of photons that our eye can see. They have very long penetration depths. 
Reaction history is the time-resolved measurement of signals emitted during an experiment. For physics experiments related to high-energy densities, plasmas, fusion energy, and fission studies, reaction history provides a time-resolved description of the fundamental physical reactions occurring during the experiment. Our current application is for ICF experiments at the Omega Laser Facility (Omega) of the University of Rochester's Lab for Laser Energetics and at the NIF. However, any experiments requiring single or sub-nanosecond time resolution for detection of x-rays, gamma rays and absolute optical timing could use the reaction history instrument.

Using reaction histories, key physics data have been taken [1], specifically the "gamma bang-time"-the onset of deuterium-tritium reactions in an ICF experiment; gamma reaction history (GRH)-the time-resolved rate of gamma production in the experiment; the x-ray reaction history-similar to the gamma reaction history; and the absolute timing of the experiment via reflected target light. The latter is the time-resolved detection of the drive laser light reflected off the experimental target. Due to jitter in the laser timing systems of Omega and NIF, and imprecision in the laser fiducial timing systems, reflected light is the only method of absolutely measuring the signal timing.

\section{OPTICAL AND OPTOMECHANICAL DESIGNS}

Gas Cherenkov detectors have been used to convert fusion gammas into photons to record gamma reaction history measurements. Gamma bang time was measured at the Omega Laser Facility (Omega) of the University of Rochester's Lab for Laser Energetics with a previously fielded system simply called the gas Cherenkov detector (GCD) [2]. This was designed by some of the Los Alamos National Laboratory developers listed for the GRH instrument; the GCD is still in use. Cherenkov radiation is the light emitted when a charged particle (in our instrument an electron) travels through a dielectric (electrically polarizable) medium (in our instrument pressurized gas) with a speed greater than that at which light would otherwise propagate in the same medium. This gas detector includes a converter, pressurized gas volume, relay collection optics, and a photon detector. The GCD is shown in Figure 3. The first successful detection of fusion gammas from DT fuel was collected at Omega in 2002. Measuring fusion gammas provide precise bang time and burn width for understanding the fusion process. The GCD uses a compact optical layout making use of a Cassegrain telescope to image the back of the converter ( $\mathrm{Be}$ or $\mathrm{Al}$ ) onto a detector. However, light is generated through the volume of gas, not just at the converter. Imaging the converter reduces collection efficiency. Some of the light is blocked by the secondary mirror structure. Because the GCD is light starved, it is placed close to the gamma source. Its position requires penetration inside the Omega laser target vacuum chamber. Because of the GCD detector's location, prompt gammas induce a precursor signal that is $0.5 \mathrm{~ns}$ ahead of the Cherenkov light. Tungsten (W) shielding reduces the amplitude of this precursor signal. But, this precursor compromises the Cherenkov signal.

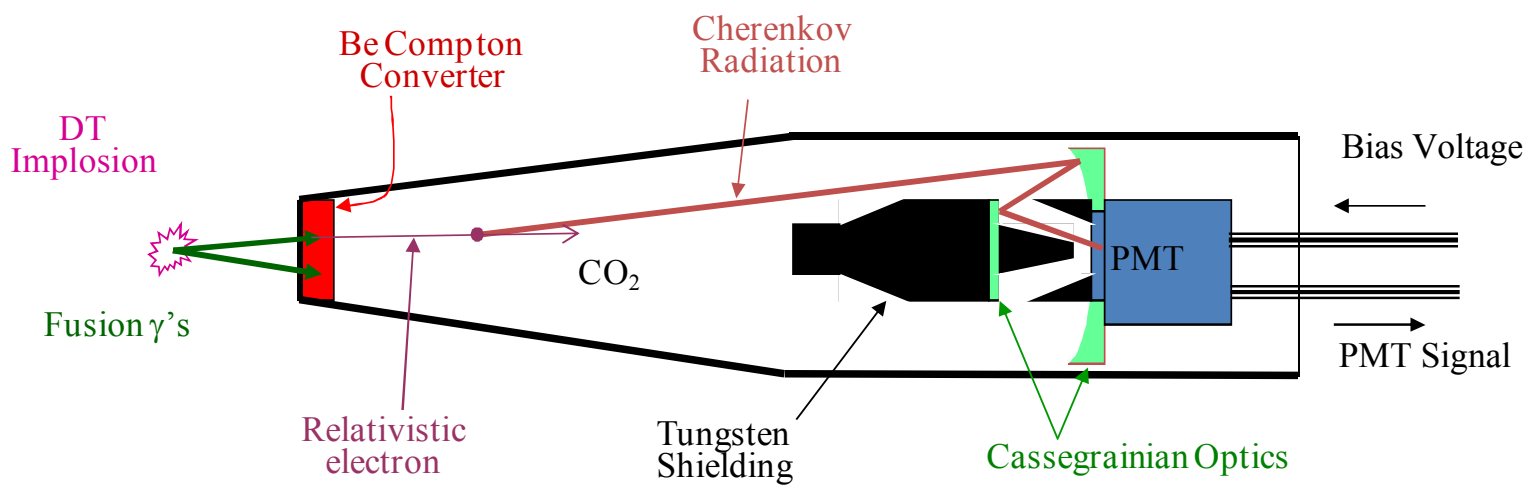

Fig. 3. Gas Cherenkov detector (GCD) fielded at LLE Omega. It is inserted inside the target vacuum chamber. Prompt gammas induce a precursor signal that is $0.5 \mathrm{~ns}$ ahead of the Cherenkov light. The tungsten shielding reduces the amplitude of this precursor signal. This GCD has been in use at Omega for almost 10 years.

For NIF a new reaction history instrument design was required. The new GRH diagnostic (shown in Figure 4) is external to the target chamber, requiring no chamber penetrations $[3,4]$. There is no requirement to position the GRH at a precise position from the gamma source. Just as in the original GCD, gammas from fusion reactions are converted to Compton electrons that are converted into Cherenkov photons (response is from 250 to $700 \mathrm{~nm}$ ) in a pressurized gas cell. Because 
of the great penetrating energy of the gammas, only about $10 \%$ of the gammas produce Compton electrons. The rest of the gammas pass through the metal components. The Cassegrain optics used in the GCD are replaced with three $90^{\circ}$ offaxis parabolic (OAP) mirrors in the GRH to relay the light into a high-speed detector. These relay optics collect light from a $125 \mathrm{~mm}$ diameter by $600 \mathrm{~mm}$ long interchangeable gas $\left(\mathrm{CO}_{2}\right.$ or $\left.\mathrm{SF}_{6}\right)$ volume. The OAP layout incorporates a 4.2 ns time delay that allows the detector to recover from prompt radiation before it records the gamma signal.

The function of the OAPs is to replace relay lenses in an optical relay system. They also function as turning mirrors that allow placement of the detector at a convenient location for shielding in a radiation environment. In Figure 4, optical ray tracings demonstrate how light can be collected from different angled trajectories of the Compton electrons as they traverse the gas volume. A Monte Carlo model of the conversion process from gammas to Cherenkov photons is used to generate photon trajectories. The collection efficiencies for different gamma energies are evaluated.

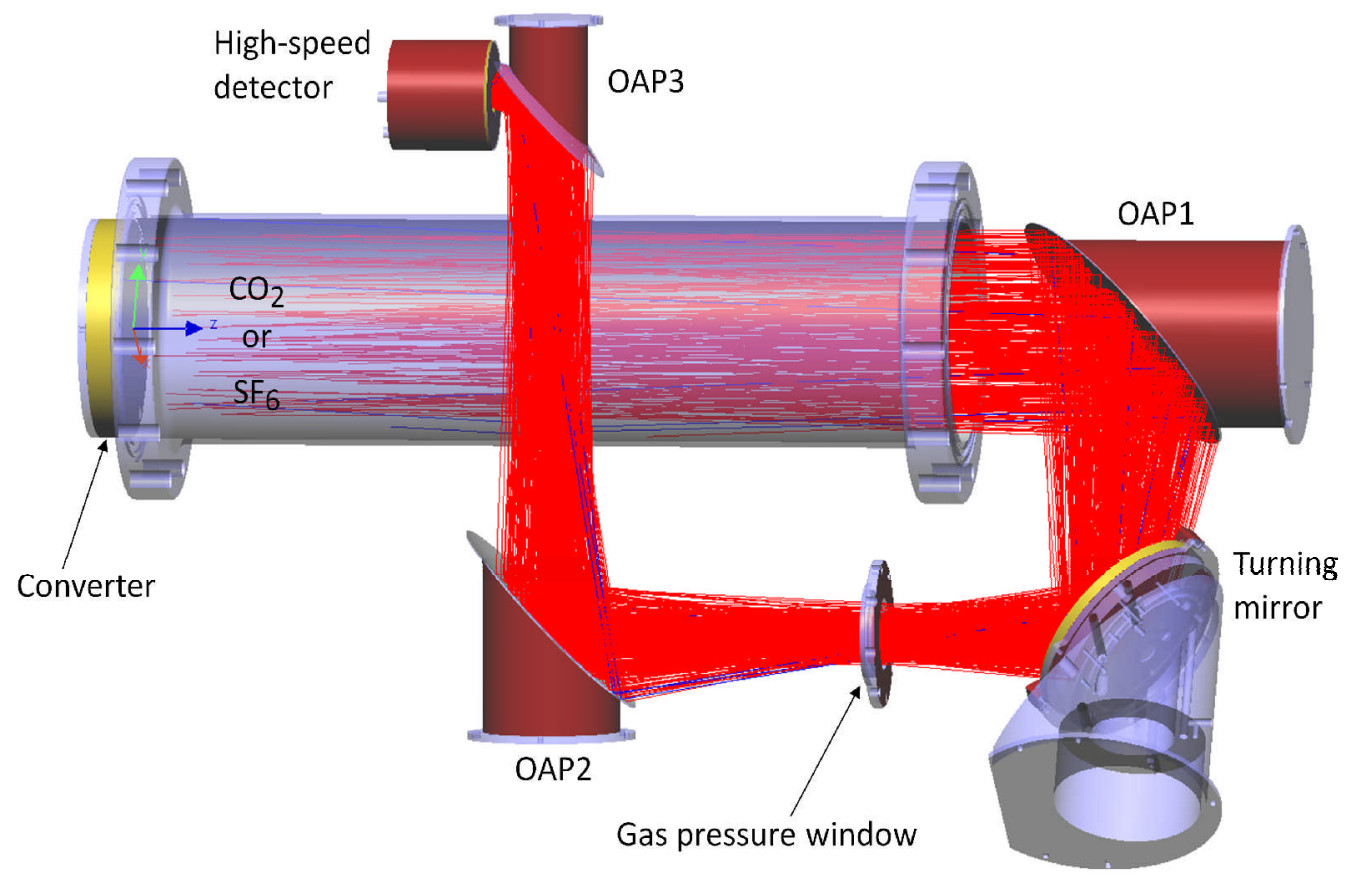

Fig. 4. Cherenkov light is generated throughout a pressurized gas cell. Monte Carlo rays derived from a simulation code show that the cell collects $5.2 \%$ of the Cherenkov light. There is ample room to surround the detector with Tungsten shielding. The turning mirror has 2 small holes allowing for optical fibers (not shown) to send calibration light towards the detector as well as towards the converter.

In our pressurized gas cell, light is collected from all source locations throughout the gas volume. The problem with using relay optics with a volume source of light is that as the object position moves, the image position also moves. However, the stop position of an imaging system never moves and its diameter does not change, as its diameter is related to the full angle cone of light collected. Thus, the stop position is the best location to place a detector that is collecting light from many object source planes. This parabolic mirror relay diagnostic is functioning as a nonimaging system.

Cherenkov light is collected into the 1-cm diameter detector as shown in Figure 5. This example ray tracing shows five gammas originating from a point at the target chamber center (TCC) located $5900 \mathrm{~mm}$ from the detector. The gammas hit five field positions on the converter, producing Compton electrons at $-2,0$, and +2 degrees. Here, Cherenkov light is emitted along each of these electron trajectories, and the detector collects light within a \pm 1 -degree cone centered about each electron trajectory. The high-speed detector has a diameter of $1 \mathrm{~cm}$. The combination of OAP2 and OAP3 are used to demagnify the first stop diameter. Due to the 10 to 1 demagnification of the first stop onto the second stop (detector), the detector collection angle is limited to \pm 3 degrees of the source light, which can be summed up from any combination of electron and photon angles (shown here are \pm 2 -degree electrons and \pm 1 -degree photons). 


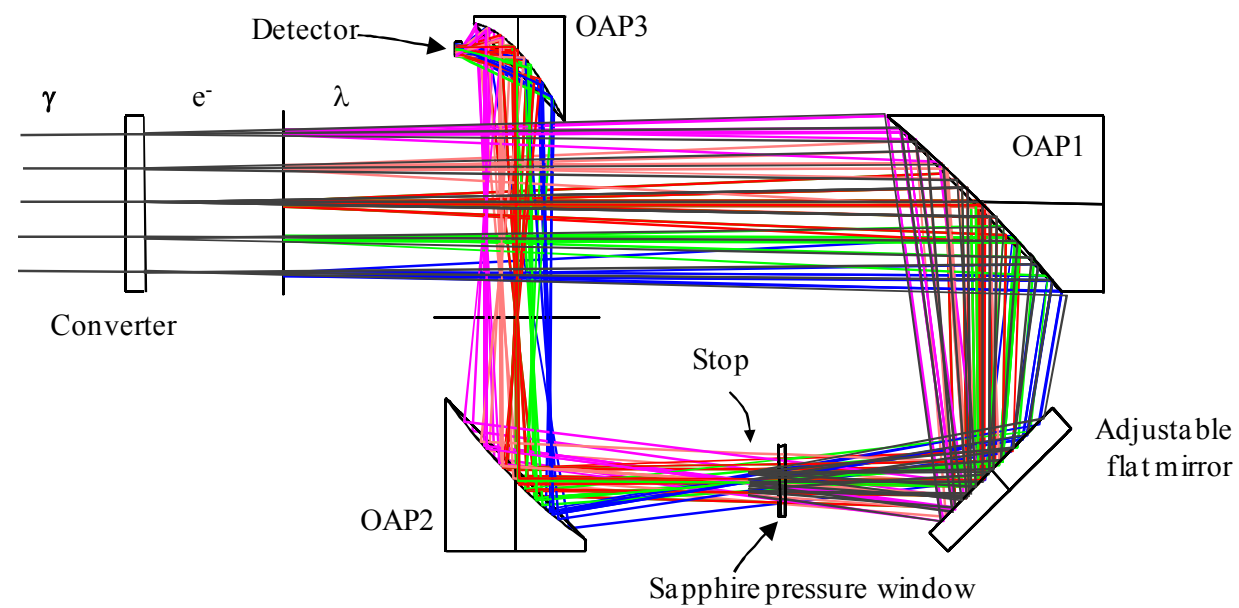

Fig. 5. Cherenkov light is generated throughout the gas cell. Shown is an optical ray tracing from one of many source planes.

The $\mathrm{Al}$ converter is $125 \mathrm{~mm}$ in diameter by $10 \mathrm{~mm}$ thick. Tungsten shielding protects both the detector and the pressure window from line-of-sight and scattered radiation. The expected burn width of DT fusion is 10-20 ps and the expected burn width of tritium-hydrogen-deuterium (THD) fusion is $80-150$ ps. The temporal dispersion produced by the sapphire pressure window is 3 ps over the wavelength range of 250 to $700 \mathrm{~nm}$. Because of the gas index, there are differences between the Compton electron and Cherenkov photons transit times that limit the optical time response of the GRH to 8 ps. Gammas will produce Cherenkov light in the sapphire pressure window, but the light travels the wrong direction due to the layout of the OAPs. The inside walls are blackened to absorb unwanted light.

Figure 6 compares the GRH design to the GCD that utilizes a Cassegrain reflector still in use at the Omega laser facility. The GCD tungsten shielding is also shown, which is seen in Figure 3. The GCD is inserted inside the Omega vacuum chamber to be up close to the gamma source. The GRH is mounted externally to the NIF vacuum chamber.

I ight is generated contimuly thromght the gas volume

shoun is only one souce plane.

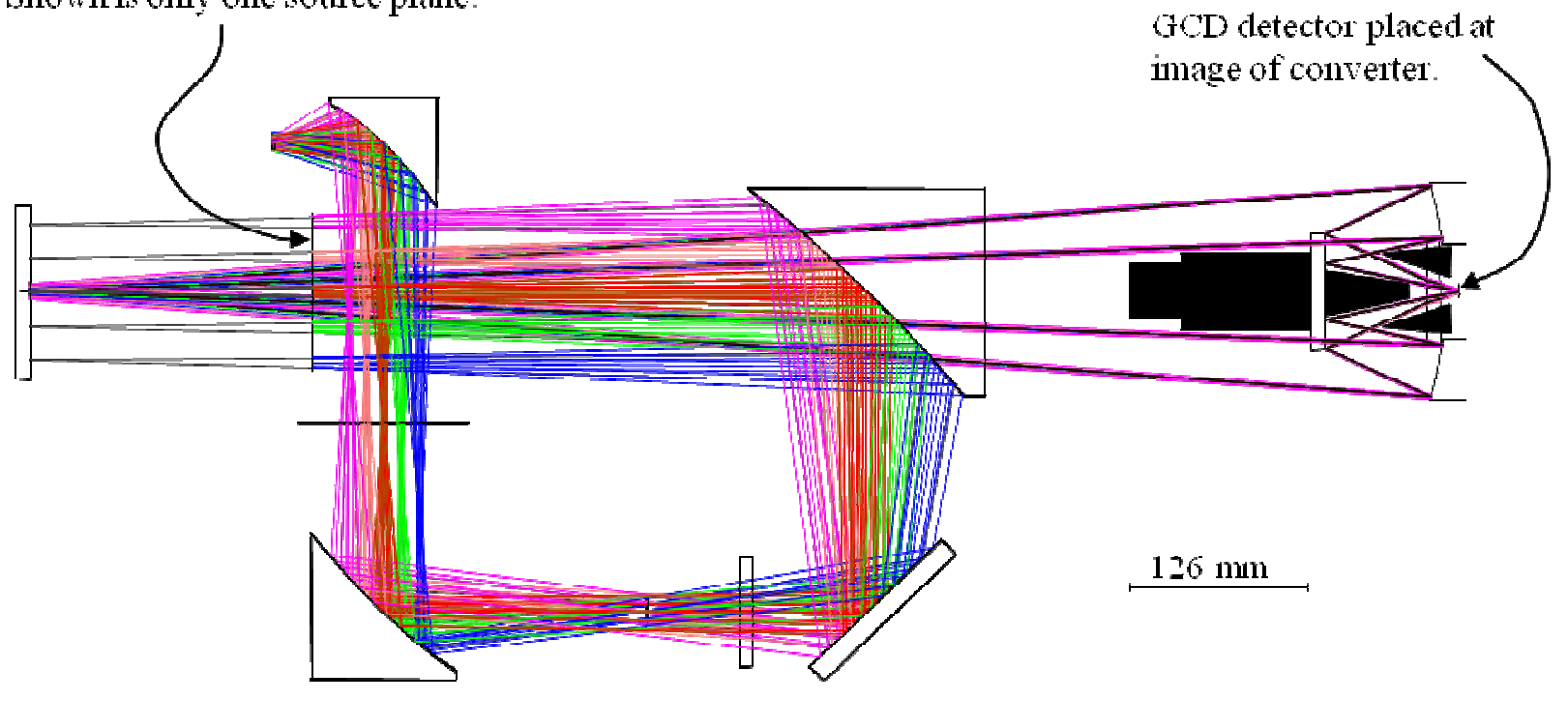

Fig. 6. GRH and GCD designs are overlaid to show size comparison and how rays are collected by their detectors.. The GRH is $3 \mathrm{X}$ more sensitive and considerably shorter. Both instruments use the same type of detector.

The GRH enclosure has no welded parts to maximize RF detector shielding. This enclosure satisfies pressure safety concerns and is shielded against electromagnetic interference induced by gammas and neutrons. Structural finite element 
analysis was done to assure that rigorous optical and safety requirements were met. Figure 7 shows labeling of the complete system. Figure 8 is a photo of the hardware mounted onto an Omega laser port.

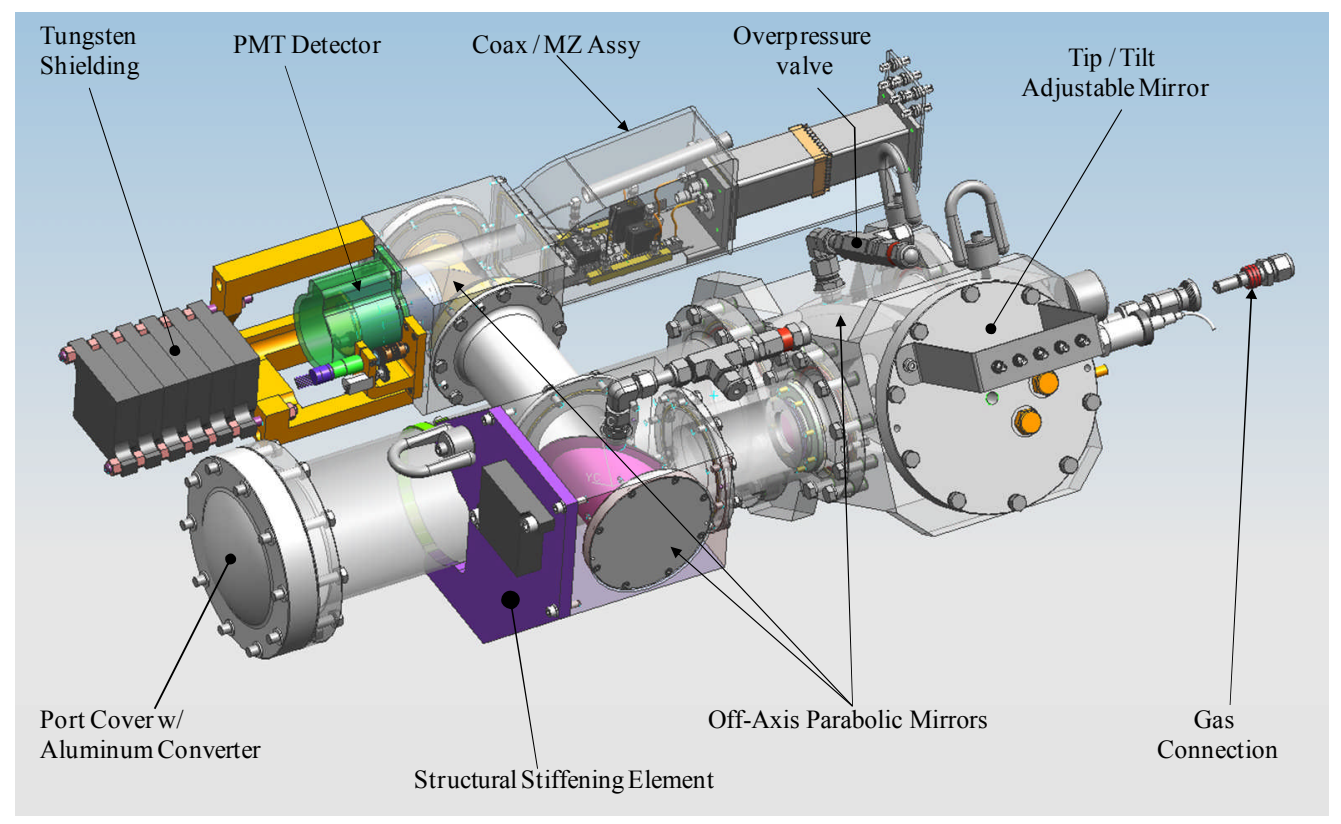

Fig. 7. Component labeling of the GRH used at LLE Omega.

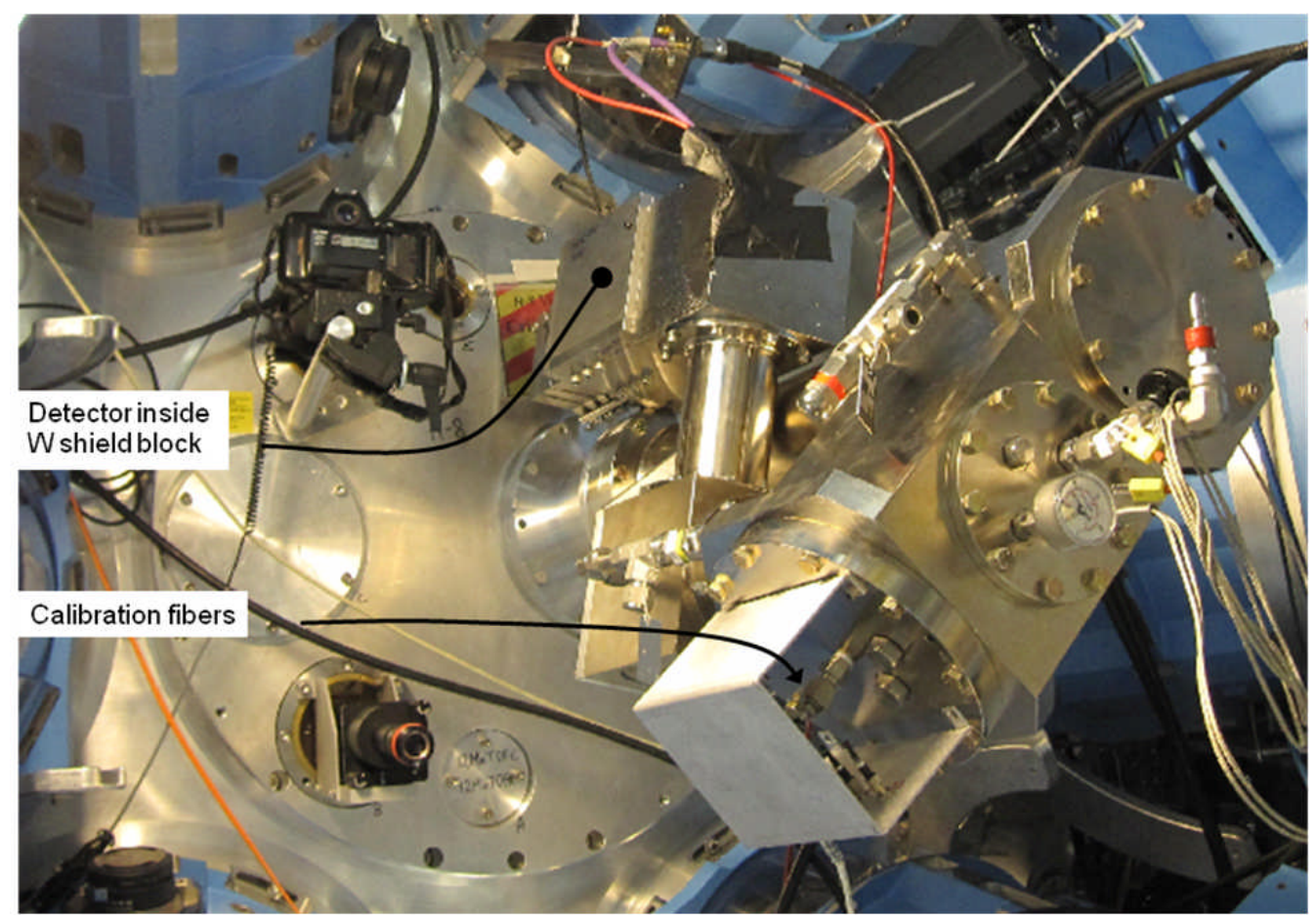

Fig. 8. The GRH mounted onto an Omega port. 
Because of the UV wavelength requirement, the parabolic mirror surface must be smooth to limit scattering. To reduce scattering of the UV light, the 90-degree off-axis parabolic mirrors are electroformed nickel mirrors instead of diamondturned aluminum mirrors $[5,6]$. For these replicated mirrors, tooling expense is applied to a master stainless steel mandrel to provide the proper surface figure and polish. Then, the mandrel is placed into a vat of nickel for hours up to days to build up the required layer of nickel. This replicated part is removed from the mandrel, coated with aluminum, and mounted onto a support structure. All mirrors are bare aluminum coated for maximum reflectivity at wavelengths below $400 \mathrm{~nm}$. Because the amount of light scattered from a surface varies as the square of the ratio of surface roughness to wavelength, diamond-turned OAPs will not work in the UV. We confirmed this formula by photographing the scattering at two different wavelengths on prototypes of both the electroformed nickel and diamond-turned mirrors. Figure 9 is a photo of OAP1 just before insertion into the GRH. Two O-ring grooves are shown, with one sealing against pressure and the other sealing against the RF.

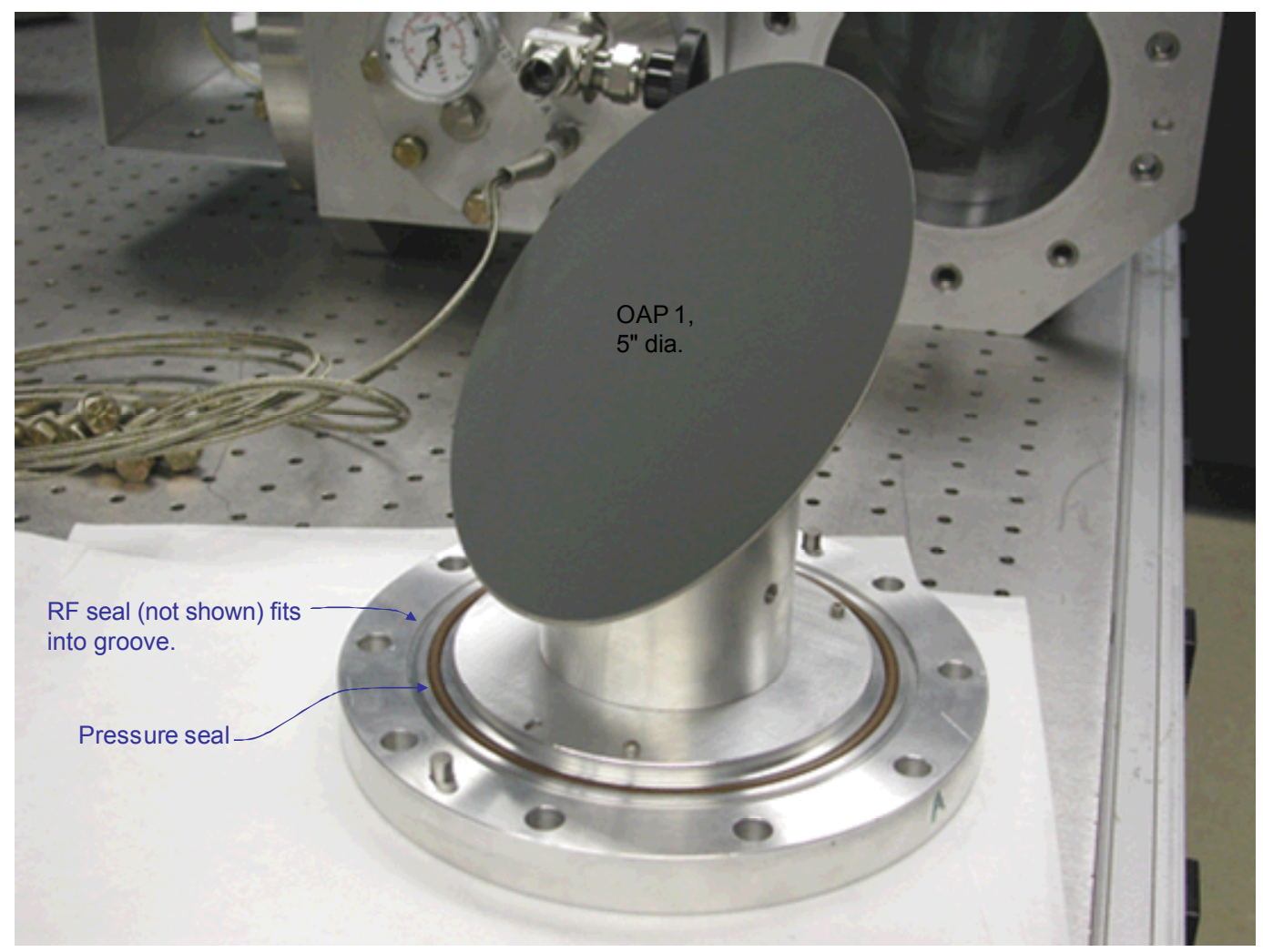

Fig. 9. Parabolic mirrors are electroformed nickel to reduce scattering of the UV light. All mirrors are bare aluminum coated for maximum reflectivity.

Aligning the optics is fairly simple. Crosshairs are placed at the converter position. The detector is replaced with a grid pattern. The turning mirror has tilt adjustments. The hole in the turning mirror is tilted to line up with the crosshairs and grid pattern, sighted by eye. If alignment is not perfect, then a different set of rays are collected at the detector, with only minor loss of sensitivity.

A single channel of the GRH is designed to work in different radiation environments. Depending on how different gamma sources are created in a dynamic environment, it may be necessary to adjust the time delay between the prompt gamma sources and the Cherenkov signals. Because the light is almost collimated between OAP2 and OAP3, their spacing can be adjusted. By changing this spacing by $\pm 150 \mathrm{~mm}$, there is very little change in the light collection seen by the detector. This results in a variable time delay of $\pm 0.5 \mathrm{~ns}$. Figure 10 shows how the detector placement can change. 


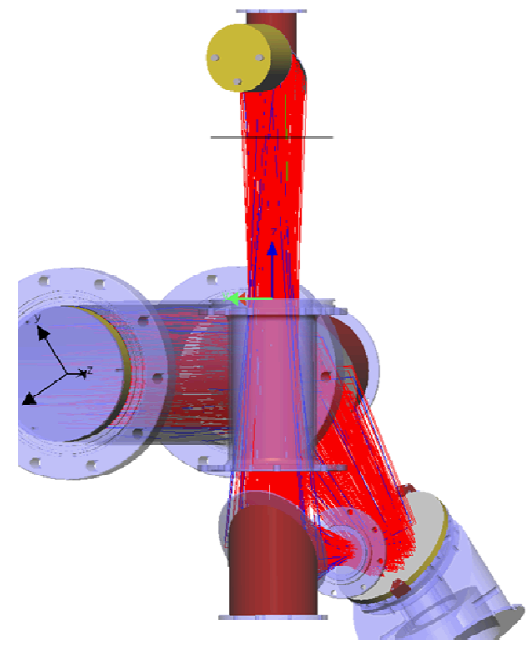

Moving OAP3 and the detector $+150 \mathrm{~mm}(0.5 \mathrm{~ns})$.

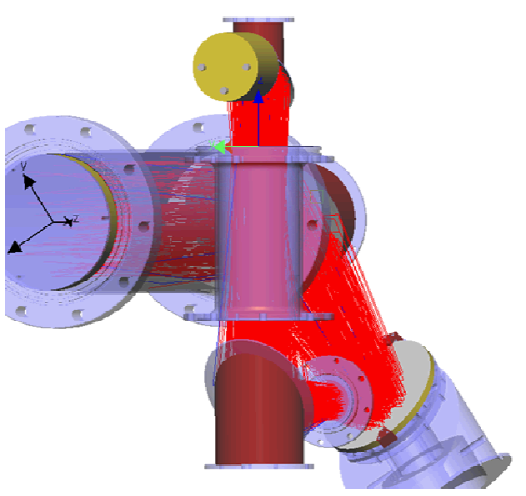

Original position of OAF3 and the detector.

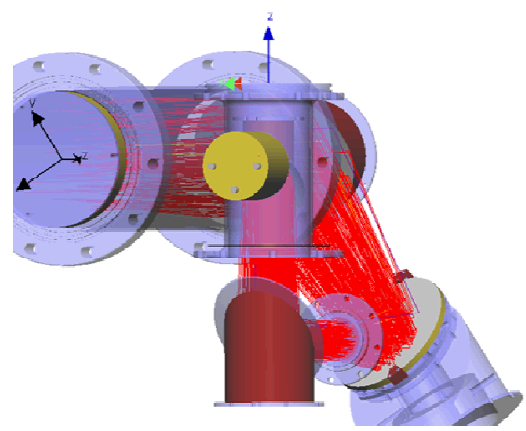

Moving OAP3 and the detector $-150 \mathrm{~mm}(-0.5 \mathrm{~ns})$.

Fig. 10. Time delay between prompt gammas and Cherenkov signals can be varied.

The GRH instrument can put many signals onto a single, absolutely timed trace as seen in Figure 11. By comparing this information carefully to the laser drive, we infer effectiveness of the laser drive, shock timing, and compression. Using the comparison to the neutron signal, we are able to do tests to determine the branching ratio of certain nuclear reactions, e.g. the gamma branching ratio of $\mathrm{He}^{3}$. Multiple reaction history lines of sight, in particular perpendicular systems, would also provide a two-dimensional areal density of the fusion fuel. Fundamentally, while reaction history is a pulsed experiment diagnostic, this same system could be adapted for use on Tokomak type fusion reactors, providing insight into sustained plasma failure modes.

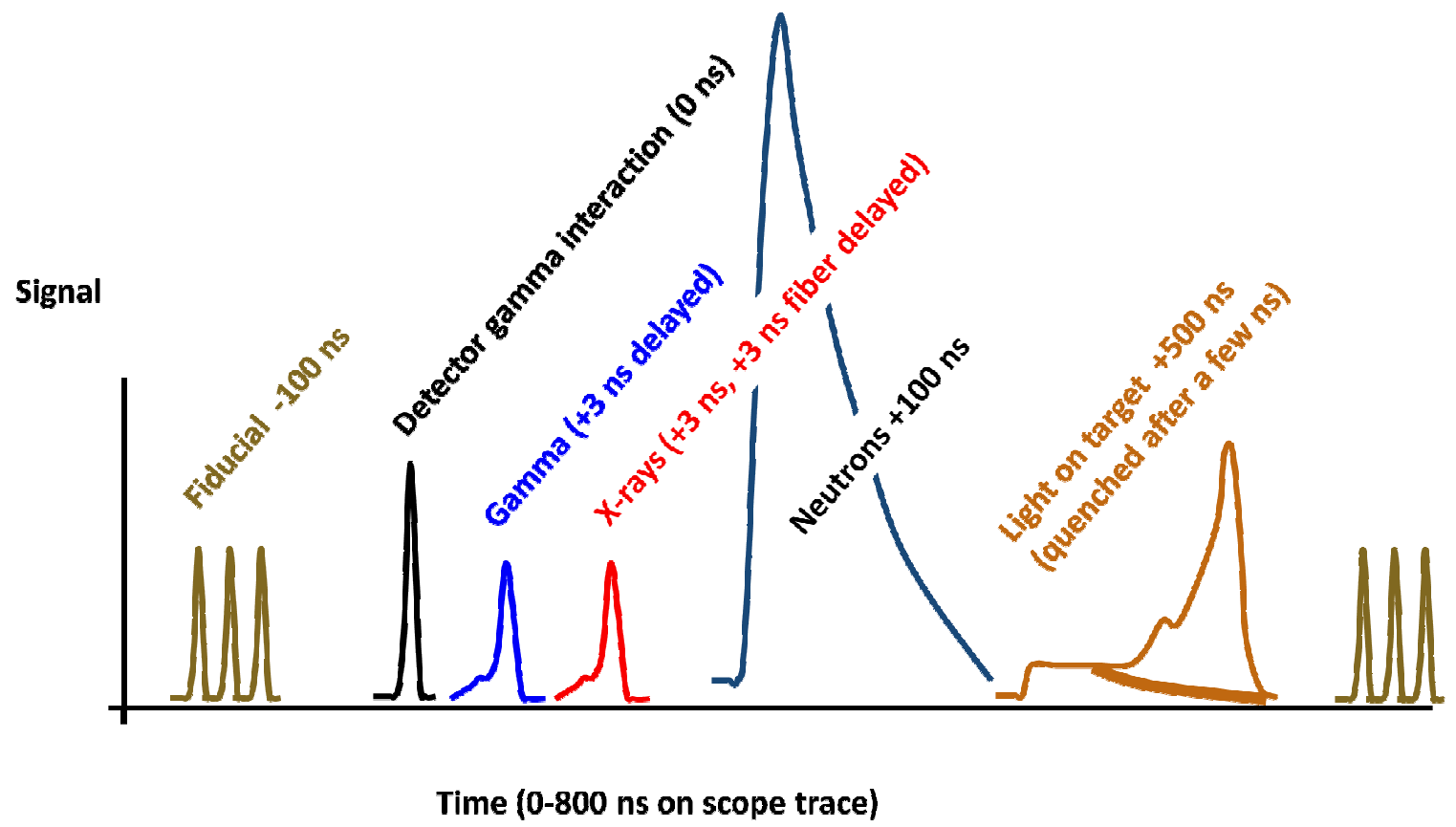

Fig. 11. The GRH data record includes cross-timing of multiple signals. Data is recorded with 3 different gain settings (e.g. $0.1,1.0,10.0)$. There is enough time spacing between the events to avoid overlap. 
At Omega different tungsten shielding arrangements were tested, stray light was measured, electromagnetic interferences were measured. At Omega, the scintillator was replaced with an $\mathrm{x}$-ray converter and the target was a gold foil. This provided an absolute time record of the GRH compared to the Omega drive lasers. Both $\mathrm{CO}_{2}$ and $\mathrm{SF}_{6}$ gas at different pressures were used. All of these criteria were validated during 2009 at the Omega laser facility during multiple shot campaigns using a single-channel system.

\section{GRH INSTALLATION ON NIF}

The initial NIF installation will have a cluster of four GRH units, each set to a different gas pressure for detecting gammas ranging from 3.5 MeV to $20 \mathrm{MeV}$. For example, $200 \mathrm{psi}$ of $\mathrm{SF}_{6}$ gives a $3.0 \mathrm{MeV}$ Cherenkov threshold, and 100 psi of $\mathrm{CO}_{2}$ gives a $6.3 \mathrm{MeV}$ Cherenkov threshold [3]. Each of the four units is attached to a support frame, as in Figure 12. This frame is attached to a NIF chamber port. The four detectors are arranged into the middle of the cluster so they experience the same shielding environment. There is an 8-inch air separation between the chamber port and converter housing. The converter can be replaced with an x-ray scintillator when recording timing signals produced by the NIF drive lasers hitting a gold target instead of a fusion capsule. The complete system attached to the port weighs $1800 \mathrm{lb}$. The design requirement for initial installation at NIF is to detect gammas emitted from fusion burns producing $3 \times 10^{13}$ to $3 \times 10^{16}$ neutrons with a system response time better than $100 \mathrm{ps}$.

Numerous recording hardware components must be calibrated and maintained. A Mach-Zehnder (MZ) modulator transmits the data by optical fibers to a remote NIF location and preserves signal bandwidth. Several high-bandwidth oscilloscopes set to different gains archive the MZ signals. Optical fibers feed light signals into each GRH unit, supplying both dry run and calibration signals. Fibers are inserted through two small holes in the flat turning mirror, as seen in Figure 13. In the current design, three fibers point towards the converter (backwards direction) to simulate a dry run signal and two fibers point towards the detector (forwards direction) to provide timing. An additional external fiber collects light from a small window at the center of this NIF port. This chamber light fiber provides an exact timing fiducial of the drive laser hitting target chamber center and, with a suitable fiber loop delay, this fiducial is added to the GRH data record.

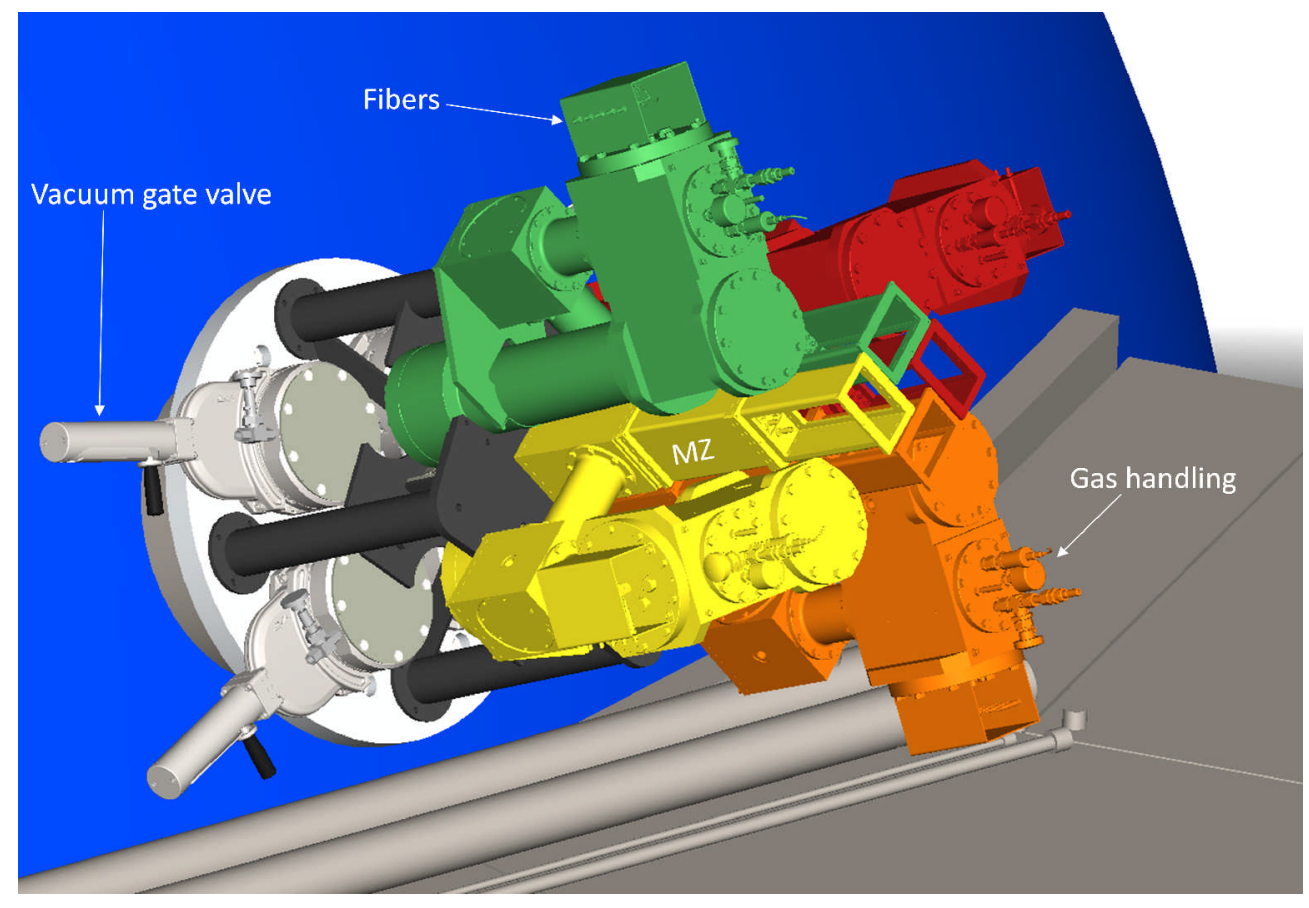

Fig. 12. NIF will have four GRH units, each with a different gas pressure, for detecting gammas at several energy ranges. The four detectors share the same shielding environment. 
Implementation at NIF will occur in two phases: (1) four photomultiplier tube (PMT)-based channels mounted to the outside of the target chamber at $\sim 6 \mathrm{~m}$ from TCC (GRH-6m) for the 1e13-1e17 DT neutron yield range expected during the early ignition-tuning campaigns; and (2) several channels located just inside the target bay shield wall at $15 \mathrm{~m}$ from TCC (GRH-15m) with optical paths leading through the cement shield wall to well-shielded streak cameras and PMTs for the 1e16-1e20 yield range expected during the DT ignition campaign. Because of the increased Cherenkov signal at $15 \mathrm{~m}$ from TCC, faster streak camera recording will provide more accurate time response of the DT fusion burn.

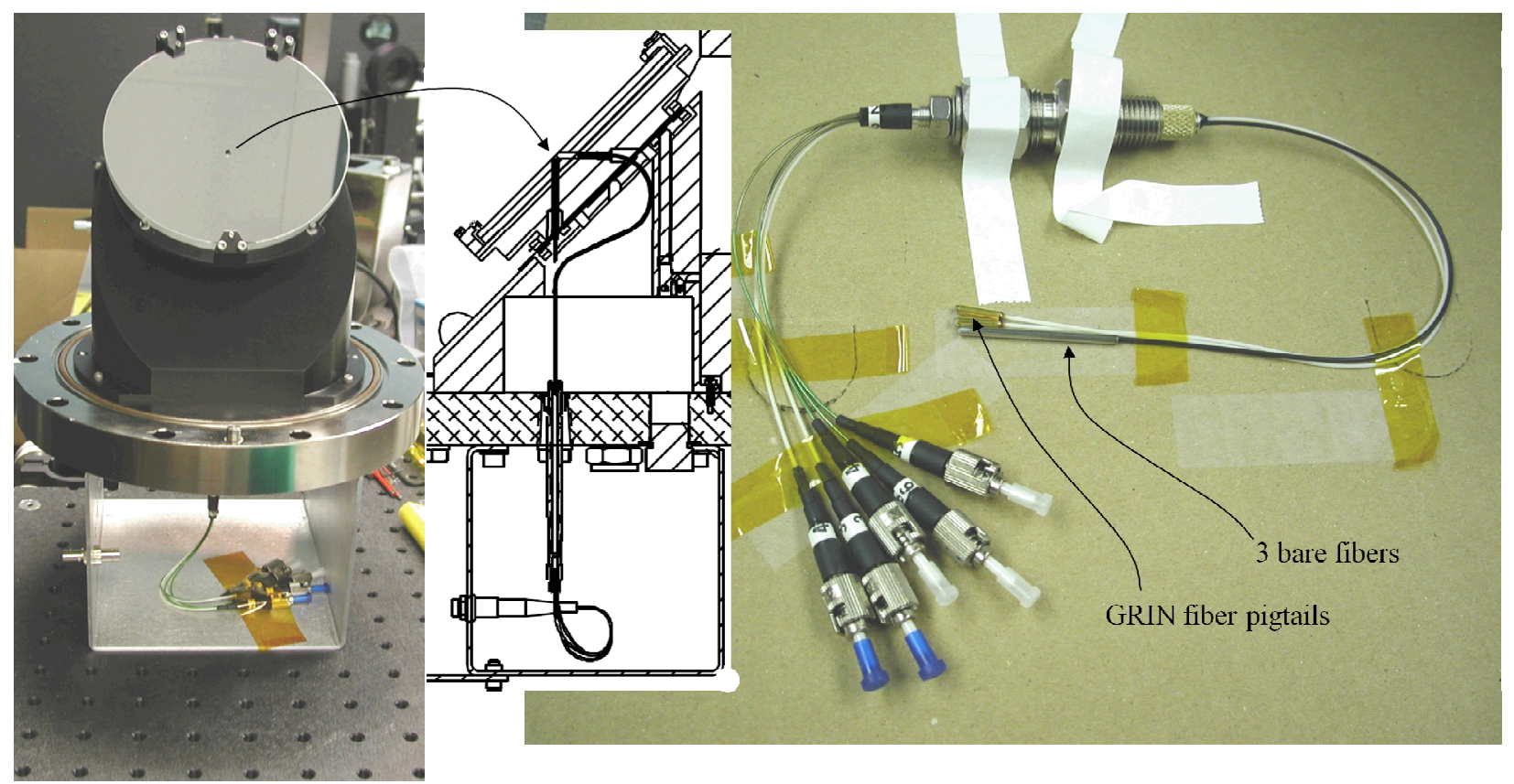

Fig. 13. Optical fibers insert light into two directions through holes in the turning mirror. Timing fiducial uses a GRIN lens fiber to point directly at the detector. Three fibers point towards the converter and simulate adry run signal.

\section{ACKNOWLEDGMENTS}

This manuscript has been authored by National Security Technologies, LLC, under Contract No. DE-AC52-06NA25946 with the U.S. Department of Energy. The United States Government retains and the publisher, by accepting the article for publication, acknowledges that the United States Government retains a nonexclusive, paid-up, irrevocable, worldwide license to publish or reproduce the published form of this manuscript, or allow others to do so, for United States Government purposes.

\section{REFERENCES}

[1] Herrmann, H. W., Young, C. S., Mack, J. M., Kim, Y. H., McEvoy, A., Evans, S. C., Sedillo, T. J., Batha, S., Schmitt, M., Wilson, D. C., Langenbrunner, J. R., Malone, R. M., Kaufman, M. I., Cox, B. C., Frogget, B. C., Miller, E. K., Ali, Z. A., Tunnell, T. W., Stoeffl, W., Horsfield, C. J. and Rubery, M., "ICF Gamma-Ray Reaction History Diagnostics," Inertial Fusion Sciences and Applications, (2009), conference proceedings to be published.

[2] Mack, J. M., Berggren, R. R., Caldwell, S. E., Evans, S. C., Faulkner, J. R., Lerche, R. A., Oertel, J. A. and Young, C. S., "Observation of high-energy deuterium-tritium fusion gamma rays using gas Cherenkov detectors," Nucl. Instrum. Methods Phys. Res. A 513, 566-572 (2003).

[3] Herrmann, H. W., Mack, J. M., Young, C. S., Malone, R. M., Stoeffl, W. and Horsfield, C. J., "Cherenkov radiation conversion and collection considerations for a gamma bang time/reaction history diagnostic for the NIF," Rev. Sci. Instrum. 79, 10E531 (2008).

[4] Malone, R. M., Herrmann, H. W., Stoeffl, W., Mack, J. M. and Young, C. S., "Gamma bang time/reaction history diagnostics for the National Ignition Facility using $90^{\circ}$ off-axis parabolic mirrors," Rev. Sci. Instrum. 79, 10E532 (2008).

[5] Opti-Forms, Inc., 42310 Winchester Rd, Temecula, CA 92590. 
[6] Kaufman, M. I., Malone, R. M., Frogget, B. C., Tunnell, T. W., Cox, B. C., Frayer, D. K., Ali, Z. A., Herrmann, H. W. and Stoeffl, W., "Optomechanical design of a prompt gamma reaction history diagnostic," in Advances in Optomechanics, Proc. SPIE 7424, 74240M (2009). 